\title{
Correlates of children's eating attitude test scores among primary school children
}

\begin{abstract}
A total of 107 Malay primary school girls (8ï $9 \mathrm{yr}$. old) completed a set of measurements on eating behavior (ChEAT, food neophobia scales, and dieting experience), the Rosenberg SelfEsteem Scale, body shape satisfaction, dietary intake, weight, and height. About $38 \%$ of the girls scored 20 and more on the ChEAT, and $46 \%$ of them reported dieting by reducing sugar and sweets (73\%), skipping meals (67\%), reducing fat foods (60\%) and snacks $(53 \%)$ as the most frequent methods practiced. In general, those girls with higher ChEAT scores tended to have lower self-esteem $(r=.39)$, indicating they were more unwilling to try new foods (food neophobic) $(r=.29)$, chose a smaller figure for desired body size $(r=-.25)$, and were more dissatisfied with their body size $(\mathrm{r}=.31)$.
\end{abstract}

Keyword: Feeding behavior; Female; Personality test; Psychological aspect; Child 